\title{
Some New Parallels Between Groups and Lie Algebras, or What Can Be Simpler than the Multiplication Table?
}

\author{
Boris KunyavskiI (Bar-Ilan University, Ramat Gan, Israel)
}

\author{
Perhaps in the times of Ahmes \\ the multiplication table was exciting.
}

Bertrand Russell ${ }^{1}$

The Greek system of numerals was very bad, so that the multiplication table was quite difficult, and complicated calculations could only be made by very clever people.

Bertrand Russell ${ }^{2}$

We give a survey of recent developments in the study of equations in groups and Lie algebras and related local-global invariants, focusing on parallels between the two algebraic structures.

\section{Foreword}

Imagine the following situation. Your kid, during the first year in elementary school, asks you to explain the notion of prime numbers (having heard about them from super-nerd classmates). Division is not yet known, only the multiplication table has been taught. What can be done? Here is a possible solution.

- Show multiplication table (see Figure 3).

- Delete the first row and the first column (corresponding to multiplication by 1 ).

- Say that prime numbers are exactly those that do not appear in such a table (add "infinitely extended" if you feel that your Wunderkind is able to understand this).

One of the goals of the present paper is to consider, in some detail, a similar situation for algebraic systems other than natural numbers, with a focus on groups and Lie algebras, with an eye towards observing some new phenomena and parallels, and in the hope of making these multiplication tables as exciting as the usual one was in the time of Ahmes and as mindchallenging as it was for ancient Greeks (see the epigraphs).

We will also consider some related problems and arising parallels, most of which are still vague and/or hypothetical.

\section{Prime elements in general algebras}

Let us write down the childlike definition of prime numbers given in the foreword in a formal fashion:

\footnotetext{
What I Believe, Kegan Paul, Trench, Trübner \& Co., London, 1925.

2 An Outline of Intellectual Rubbish: A Hilarious Catalogue of Organized and Individual Stupidity, Haldeman-Julius Publications, Girard, Kansas, 1943.
}

Definition 2.1. Let $A=\mathbb{N} \backslash\{1\}=\{2,3,4,5, \ldots\}$. Equip $A$ with usual multiplication. Then $a \in A$ is prime if the equation $x y=a$ has no solutions $(x, y) \in A \times A$.

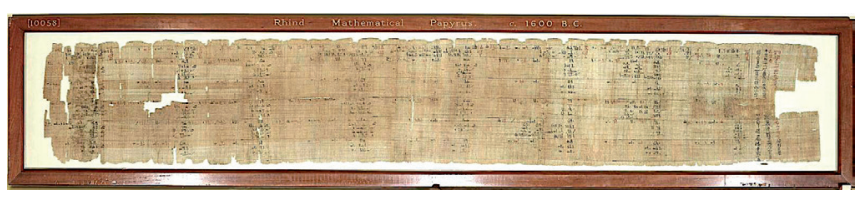

Figure 1. "Rhind Mathematical Papyrus", around 1550 BC (British Museum reference: EA10058 @ The Trustees of the British Museum)

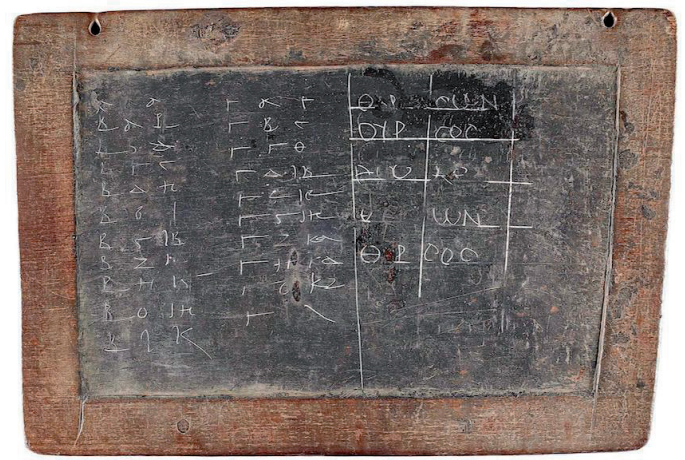

Figure 2. Greek Multiplication table on a wax tablet (British Museum AddMS34186. About 100AD. (C) The Trustees of the British Museum)

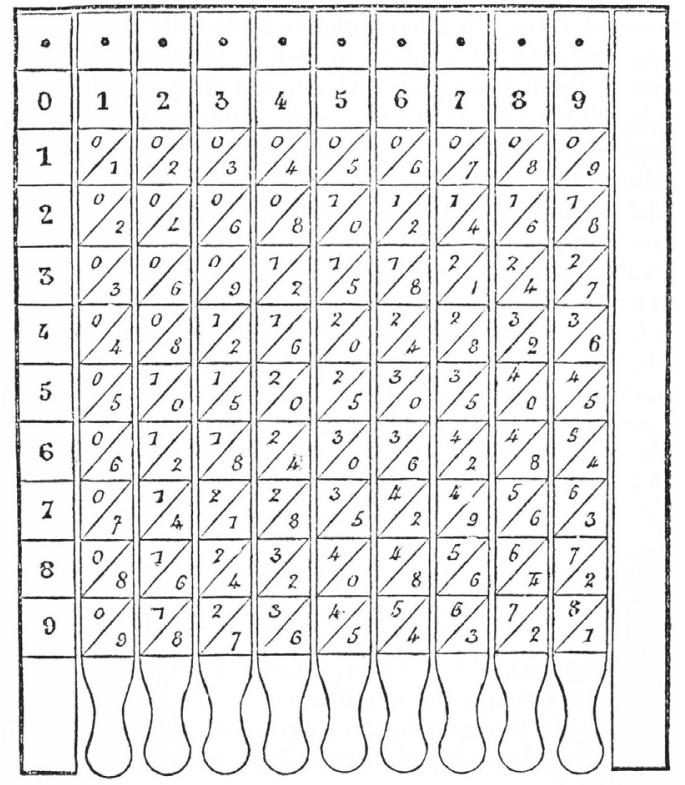

Figure 3. Multiplication table (Wikimedia Commons, originally published in: Popular Science Monthly, volume 26, 1885, p. 451) 
Let us now mimic it and extend it to arbitrary algebras.

Definition 2.2. Let $A$ be an algebra equipped with a binary operation (the sign of which will be omitted). Then we say that $a \in A$ is prime if the equation $x y=a$ has no solutions $(x, y) \in A \times A$.

Example 2.3. (Group commutators) Let $G$ be a group, and let $A_{G}$ be the underlying set of $G$ with operation $[x, y]:=$ $x y x^{-1} y^{-1}$. For brevity, let us call prime elements of the algebra $A_{G}$ prime elements of $G$. These are elements of $G$ not representable as a single commutator.

Denote by $[G, G]$ the subgroup of $G$ generated by all commutators $[x, y], x, y \in G$, and recall that $G$ is said to be perfect if $[G, G]=G$.

If $G$ is not perfect, it obviously contains prime elements: any $a \notin[G, G]$ is prime.

\section{$3 \quad$ Wide groups}

Definition 3.1. We say that a group $G$ is wide if $[G, G]$ contains prime elements.

Historically, the first example of a wide group (of order 1024) was attributed to George Abram Miller (see [31]). In 1977, in his $\mathrm{PhD}$ thesis, Robert Guralnick proved that the smallest wide group is of order 96 (see [39]). Actually, there are two non-isomorphic wide groups of order 96. Nowadays, such statements can easily be verified by computer. The smallest wide perfect group is of order 960. Further examples and results can be found in a survey paper by Luise-Charlotte Kappe and Robert Morse [50] and in the Bourbaki 2013 talk delivered by Gunter Malle [62].

The next step, from perfect to simple groups, is far more tricky. The cases of finite and infinite groups should be considered separately.

In the case where $G$ is finite, each element is a single commutator. This was conjectured by $\varnothing$ ystein Ore in the 1950s [70]. The proof required lots of various techniques. Most groups of Lie type were treated by Erich Ellers and Nikolai Gordeev in the 1990s [26]. The proof was finished by Martin Liebeck, Eamonn O'Brien, Aner Shalev and Pham Huu Tiep in 2010 [60]. See Malle's Bourbaki talk [62] for details.

If $G$ is infinite, the situation is entirely different.

There are several cases where each element of $G$ is a single commutator: $G=A_{\infty}$ is the infinite alternating group (Ore [70]); $G=\mathcal{G}(k)$ is the group of $k$-points of a semisimple adjoint linear algebraic group $\mathcal{G}$ over an algebraically closed field $k$ (Rimhak Ree [74]); and $G$ is the automorphism group of some "nice" topological or combinatorial object (e.g., the Cantor set). Precise references and additional examples and generalisations can be found in our survey [48] (jointly with Alexey Kanel-Belov and Eugene Plotkin).

The first example of the opposite kind was discovered by Jean Barge and Étienne Ghys in 1992 [5]. Looking at the title of their paper, it is hard to suspect that it is about the multiplication table in groups. Indeed, the group they constructed is of differential-geometric origin. It is simple and wide (it contains elements not representable as a single commutator). Both statements are proved using highly nontrivial geometrical arguments. Later on, more examples of such a kind were constructed (Alexey Muranov [67], Pierre-Emmanuel Caprace and Koji Fujiwara [21], Elisabeth Fink and Andreas Thom [30]).

These groups are indeed very different from the "nice" groups discussed above. For any group $G$, one can introduce the following notions.

For any $a \in[G, G]$ define its length $\ell(a)$ as the smallest number $k$ of commutators needed to represent it as a product $a=\left[x_{1}, y_{1}\right] \ldots\left[x_{k}, y_{k}\right]$. Define the commutator width of $G$ as $\operatorname{wd}(G):=\sup _{a \in[G, G]} \ell(a)$. It turns out that for a simple group $G$, the commutator width $\operatorname{wd}(G)$ may be as large as we wish, or even infinite (such examples appear in the papers by Barge-Ghys and Muranov).

\section{First parallels: Wide Lie algebras}

Now let $L$ be a Lie algebra defined over a field $k$. As above, we say that $L$ is wide if the derived algebra $[L, L]$ contains elements which are not representable as a single Lie bracket. As in the case of groups, wide Lie algebras naturally appear among finite-dimensional nilpotent Lie algebras (see, e.g., the MathOverflow discussion [63]). It is worth noting that the smallest dimension where such examples arise is 10, which is parallel to Miller's example of a wide group of order $1024=2^{10}$ mentioned above. This phenomenon is not surprising, in light of well-known relations between nilpotent groups and Lie algebras: they go back to the classical Baker-Campbell-Hausdorff formula and culminate in the correspondence of categorical flavour, due to Anatoly Mal'cev and Michel Lazard (see, e.g., the monograph of Evgenii Khukhro [51] for some details concerning this correspondence). With some effort one can also construct wide perfect Lie algebras (see [7]). We shall focus on the case of simple Lie algebras. Here are our main questions.

Question 4.1. Does there exist a wide simple Lie algebra?

More generally, as in the case of groups, one can define for every $a \in[L, L]$ its bracket length $\ell(a)$ as the smallest $k$ such that $a$ is representable as a sum $a=\left[x_{1}, y_{1}\right]+\cdots+\left[x_{k}, y_{k}\right]$, and then define the bracket width of $L$ as $\operatorname{wd}(L):=\sup _{a \in[L, L]} \ell(a)$.

If Question 4.1 is answered in the affirmative, one can ask the next question:

Question 4.2. Does there exist a simple Lie algebra $L$ of $i n-$ finite bracket width?

Where does one look for counter-examples? Below $L$ is a simple Lie algebra over a field $k$.

First suppose that $L$ is finite-dimensional. In the following cases it is known that every element is a single bracket (i.e., $\operatorname{wd}(L)=1$ ): $L$ is split and $k$ is sufficiently large (Gordon Brown [14]; Ralf Hirschbühl [41] improved estimates on the size of $k$ ); $k=\mathbb{R}, L$ is compact (there are many different proofs, attributed to Dragan Đoković and Tin-Yau Tam [25], Karl-Hermann Neeb (see [42]), Dmitri Akhiezer [1], Alessandro D'Andrea and Andrea Maffei [22], Joseph Malkoun and Nazih Nahlus [61]); some non-compact algebras $L$ over $\mathbb{R}$ (Akhiezer, op. cit.).

The most interesting unexplored class in finite-dimensional case is the family of algebras of Cartan type over a 
field of positive characteristic. As a working hypothesis, one can suspect that none of these algebras are wide.

Remark 4.3. If $L$ is finite-dimensional over any infinite field of characteristic different from 2 and 3, its bracket width is at most two (George Bergman and Nazil Nahlus [7]).

Suppose now that $L$ is infinite-dimensional. There are several natural families of simple infinite-dimensional Lie algebras. Here are some of them: four families $W_{n}, H_{n}, S_{n}, K_{n}$ of algebras of Cartan type; (subquotients of) Kac-Moody algebras; algebras of vector fields on smooth affine varieties.

As to the first case, a theorem by Alexei Rudakov [77] shows that none of the algebras $L$ of Cartan type are wide (we owe this observation to Zhihua Chang). I am unaware of any approach to the second family, though there are simple KacMoody groups of infinite width (see the paper by Caprace and Fujiwara [21] mentioned above).

Question 4.4. Are there wide simple Kac-Moody algebras?

Even the case of affine Kac-Moody algebras seems open.

However, the third family, which moves us back to the origin of the area, turned out to be more promising. Actually, algebras of vector fields appeared in the work of the founders of the theory, Sophus Lie and Élie Cartan.

In our work in progress [57] (joint with Andriy Regeta) we established the following fact.

Theorem 4.5. Among Lie algebras of vector fields on smooth affine varieties there are wide algebras.

Some details are in order.

Let $k$ be an algebraically closed field of characteristic zero. Let $X \subset \mathbb{A}_{k}^{n}$ be an irreducible affine $k$-variety. Let $\operatorname{Vect}(X)$ denote the collection of (polynomial) vector fields on $X$, i.e., $\operatorname{Vect}(X)=\operatorname{Der}(O(X))$, the set of derivations of the algebra of regular functions on $X$. It carries a natural structure of Lie algebra, as a Lie subalgebra of the algebra of endomorphisms $\operatorname{End}_{k}(O(X))$ :

$$
[\xi, \eta]:=\xi \circ \eta-\eta \circ \xi .
$$

There are strong relations between properties of $X$ and $\operatorname{Vect}(X)$ We only mention a couple of the most important facts: two normal affine varieties are isomorphic if and only if $\operatorname{Vect}(X)$ and $\operatorname{Vect}(Y)$ are isomorphic as Lie algebras (Janusz Grabowski [37] for smooth varieties, Thomas Siebert [87] in general); $X$ is smooth if and only if $\operatorname{Vect}(X)$ is simple (David Alan Jordan [46], Siebert, op. cit.; see also the lecture notes by Hanspeter Kraft [53] and a new proof by Yuli Billig and Vyacheslav Futorny [8]).

Example 4.6. Let $X=\mathbb{A}^{n}$. Then $L=\operatorname{Vect}\left(\mathbb{A}^{n}\right)$ is a free $O\left(\mathbb{A}^{n}\right)=k\left[x_{1}, \ldots, x_{n}\right]$-module of rank $n$ generated by $\partial_{x_{i}}=$ $\frac{\partial}{\partial x_{i}}, i=1, \ldots, n$. It is an easy exercise to show that every element of $L$ can be represented as a single Lie bracket. We leave the proof to the reader.

The situation is not as simple for more general affine varieties, even for curves. The following example, by Billig and Futorny [8], shows the essence of the problem.

Example 4.7 ([8]). Let $H=\left\{y^{2}=2 h(x)\right\}$ where $h(x)$ is a separable monic polynomial of odd degree $2 m+1 \geq 3, A=$
$O(H)=k[x, y] /\left\langle y^{2}-2 h(x)\right\rangle, D=\operatorname{Vect}(H)=\operatorname{Der}_{k}(A)$. Then $D$ is a free $A$-module of rank 1 generated by $\tau=y \partial_{x}+h^{\prime}(x) \partial_{y}$. The algebra $D$ contains neither semisimple nor nilpotent elements. (We say that $\eta \in D$ is semisimple if $\operatorname{ad}(\eta)$ has an eigenvector.)

\section{Theorem 4.8 ([57]). The Lie algebra D is wide.}

Idea of proof. One can introduce a filtration on $D$ so that the smallest nonzero degree is $2 m-1$. Then any $\eta \in D$ with $\operatorname{deg} \eta=2 m-1$ is not representable as a single Lie bracket.

Here is another example for the case of surfaces.

Example 4.9. Let $S=\{x y=p(z)\} \subset \mathbb{A}_{k}^{3}$ where $p(z)$ is a separable polynomial, $\operatorname{deg} p \geq 3$ (Danielewski surface). Let $L=L N D(S)$ be the subalgebra of $\operatorname{Vect}(S)$ generated by all locally nilpotent vector fields.

Lemma 4.10 (Matthias Leuenberger and Andriy Regeta [59]). L is a simple Lie algebra.

Assuming that the surface satisfies a certain additional condition on the Jacobian of regular functions, we can prove the following fact.

Theorem 4.11 ([57]). L is a wide Lie algebra.

The proof is based on the same paper by Leuenberger and Regeta [59] and uses degree arguments.

Question 4.12. What is the bracket width of the algebras $\operatorname{Vect}(H)$ and $L N D(S)$ ?

Here are some further questions.

- What geometric properties of $X$ are responsible for the fact that the Lie algebra $\operatorname{Vect}(X)$ is wide?

- Does there exist a Lie-algebraic counterpart of the BargeGhys example? This requires going over to the category of smooth vector fields on smooth manifolds.

- Where should one look for further examples of wide simple Lie algebras?

There are two candidates, both suggested by Yuli Billig.

Let $K_{2}$ denote the Lie algebra obtained from the matrix

$$
\left(\begin{array}{ll}
2 & 2 \\
2 & 2
\end{array}\right)
$$

in the same way as Kac-Moody Lie algebras are obtained from generalised Cartan matrices, see the seminal paper of Victor Kac [47]. Is $K_{2}$ wide?

Further eventual examples could be found among the most natural generalisation of examples of Theorem 4.8, in the class of algebras of Krichever-Novikov type (see, e.g., the monograph by Martin Schlichenmaier [80]). One can ask whether there are wide simple algebras of Krichever-Novikov type. If yes, can the width be arbitrarily large? Can it be infinite?

One can ask a 'metamathematical' question.

Question 4.13. Let $L$ be a 'generic' ('random', 'typical') simple Lie algebra. Is $L$ wide?

Of course, any eventual answer will heavily depend on what is meant by 'random', 'typical', etc. However, the absence of semisimple and nilpotent elements in the Lie algebra $\operatorname{Vect}(H)$ mentioned above is a witness of the absence of 
any analogue of the triangular decomposition. This is in sharp contrast with the situation for Kac-Moody algebras and gives some evidence for the following ('metamathematical') working hypothesis.

Less structured ('amorphous') Lie algebras tend to be wide.

Informally, these opposite hypotheses can be illustrated by the difference between the skeletons of fish and jellyfish (yes, jellyfish do have skeletons).

\section{$5 \quad$ Word equations in groups and polynomial equations in Lie algebras}

Let us now present a wider perspective on the notions discussed in the previous section. Namely, suppose we are given a group $G$ with operation $[g, h]=g h g^{-1} h^{-1}$ (resp. a Lie algebra $L$ with bracket [, ]). Trying to find a given element $a$ of $G$ (resp. of $L$ ) in its multiplication table, we search for a solution of the equation

$$
[x, y]=a
$$

in $G \times G$ (resp. in $L \times L$ ), i.e., the right-hand side is fixed and $x, y$ are unknowns.

One can generalise equation (1) as follows.

Let $w(x, y)$ denote a group word in $x, y$ (more formally, an element of the free group $\mathcal{F}_{2}=\mathcal{F}(x, y)$ ). One may think of something like $x^{2} y^{2020} x^{-1} y^{-3}$. Even more generally, for any integer $d \geq 1$ one can consider $w\left(x_{1}, \ldots, x_{d}\right) \in \mathcal{F}_{d}=$ $\mathcal{F}\left(x_{1}, \ldots, x_{d}\right)$ and for every group $G$ and $a \in G$ look for solutions of the word equation

$$
w\left(x_{1}, \ldots, x_{d}\right)=a .
$$

In a similar way, one can consider a Lie polynomial $P\left(X_{1}, \ldots, X_{d}\right)$ (an element of the free Lie algebra $\mathcal{L}_{d}=$ $\left.\mathcal{L}\left(X_{1}, \ldots, X_{d}\right)\right)$ and for every Lie algebra $L$ and $A \in L$ look for solutions of the equation

$$
P\left(X_{1}, \ldots, X_{d}\right)=A \text {. }
$$

Remark 5.1. Note that our set-up only includes equations with constant-free left-hand side. This means that if, say, $A, B$ are fixed elements of a Lie algebra $L$, we consider equations $[X, Y]=A$ but not $[B, X]=A$. As to equations with constants, see our joint papers with Nikolai Gordeev and Eugene Plotkin [32]-[34], the paper by Anton Klyachko and Andreas Thom [52], and the references therein. Also, to avoid any confusion, we want to emphasise that in our set-up, solutions of (2) are sought in $G$, and not in an overgroup of $G$.

Here are some natural questions one can ask about equation (2) (of course, similar questions arise for equation (3)).

Question 5.2. Let a group $G$ be given. Is equation (2) solvable

(a) for all $a \in G$, or, at least,

(b) for a 'typical' $a \in G$ ?

Part (a) leads to a natural generalisation of the notion of commutator width of $G$ discussed in Section 3 (the so-called $w$-width). Various situations where one can guarantee that part (a) is answered in the affirmative are described in some detail in the monograph by Dan Segal [81] and in several survey papers; apart from [48] mentioned above, see also the papers by Aner Shalev [82, 83], and our more recent papers [2] (jointly with Tatiana Bandman and Shelly Garion) and [35] (jointly with Nikolai Gordeev and Eugene Plotkin).

As to part (b), any change in a precise definition of 'typical' may be critically important for an eventual answer. Say if $G$ can be equipped with different topologies and 'typical' translates as 'belonging to a dense set in the chosen topology', the answer to (b) heavily depends on this choice. Here is an archetypical example where this dependence is the most striking: $G$ is (the group of rational points of) a linear algebraic group defined over a field $k$. For this class of groups, Armand Borel established a general result. To formulate it, it is convenient to introduce the following notion.

Definition 5.3. Let $d$ be a positive integer, and let $w=$ $w\left(x_{1}, \ldots, x_{d}\right) \in \mathcal{F}_{d}$ be a word. For a group $G$ define a map

$$
w: G^{d} \rightarrow G
$$

by evaluation: $\left(g_{1}, \ldots, g_{d}\right) \mapsto w\left(g_{1}, \ldots, g_{d}\right)$.

Such maps will be called word maps. If $G$ is non-abelian, $w$ is not a group homomorphism. In some special cases, one can say more about these maps. Let $\mathcal{G}$ be a linear algebraic group defined over a field $k$. Then, given a word $w \in \mathcal{F}_{d}$, one can define a morphism of the underlying algebraic $k$-varieties $w: \mathcal{G}^{d} \rightarrow \mathcal{G}$ which induces the word map (4) on the group $G=\mathcal{G}(k)$ of $k$-points of $\mathcal{G}$ (and, more generally, on $K$-points $\mathcal{G}(K)$ for any field extension $K / k$ ). We denote all these maps by the same letter $w$ with the hope that this does not cause any confusion.

Theorem 5.4 (Borel [11]). Let $\mathcal{G}$ be a connected semisimple linear algebraic group defined over a field $k$. Then for any non-identity word $w \in \mathcal{F}_{d}$, the morphism $w: \mathcal{G}^{d} \rightarrow \mathcal{G}$ is dominant.

Recall that this means that the image of $w$ contains a Zariski-dense open set (or, informally, that equation (2) with a 'typical' right-hand side is solvable).

Remark 5.5. It is worth emphasising the role of Zariski topology in this statement. One should not think that the assertion remains true in any topology. Andreas Thom [89] noticed that for the special unitary group $\mathcal{G}=S U_{n}$, word maps on the compact group $G=\mathcal{G}(\mathbb{R})$, equipped with the Euclidean topology (some people, especially those who are far removed from algebraic geometry, call it 'natural'), may behave quite differently. Namely, given $\epsilon>0$, one can find a word $w \in \mathcal{F}_{2}$ such that the image of the word map (4) is contained in the open disk of radius $\epsilon$ centred at the identity matrix. (There is no contradiction with Borel's theorem because such a disk is Zariski-dense.)

Remark 5.6. One cannot expect to extend Borel's theorem too far beyond the class of semisimple groups, see [35] for some argumentation. Perhaps the only general hope is to treat perfect algebraic groups. Under certain additional assumptions, the dominance statement has been established in [34], and one has no examples of perfect algebraic groups $G$ and words $w$ for which the word morphism is not dominant.

\section{Towards infinitesimal analogues}

One can consider infinitesimal analogues of the problems discussed in the previous section. Namely, for a Lie polynomial 
$P\left(X_{1}, \ldots, X_{d}\right)$ one can ask the following question, similar to Question 5.2:

Question 6.1. Let a Lie algebra $L$ be given. Is equation (3) solvable

(a) for all $A \in L$, or, at least,

(b) for a 'typical' $A \in L$ ?

As in the group case, it is convenient to introduce the corresponding evaluation map:

$$
P: L^{d} \rightarrow L, \quad\left(a_{1}, \ldots, a_{d}\right) \mapsto P\left(a_{1}, \ldots, a_{d}\right) .
$$

In these terms, Question 6.1 can be rephrased as the question about the surjectivity or dominance of the map (5).

In view of Remark 5.6, it is reasonable to focus on simple Lie algebras.

Let us take an informal look at the known ways to go over from groups to Lie algebras (or in the opposite direction).

Let us briefly recall several classical approaches to such a transition, without pretending to give a comprehensive overview.

First bridges between groups and Lie algebras had been built even before these notions were defined in a formal way. One can mention Poisson and Jacobi, whose pioneering works on Hamiltonian mechanics paved a road towards what is nowadays called Poisson geometry, Poisson-Lie groups, etc. (see a nice survey by Alan Weinstein [91] for details).

One can also mention Arthur Cayley, whose ingenious formula, allowing one to pass from special orthogonal to skew-symmetric matrices, was the first instance of what is now called an equivariant birational isomorphism between an algebraic group and its Lie algebra; see the books by Hermann Weyl [92] and Mikhail Postnikov [72] for a detailed discussion and further development of this idea. Note that like the work of Poisson-Jacobi, Cayley's invention served as a tool in theoretical mechanics (this time the Lagrangian variant). Note also that the limits up to which the Cayley transform can be generalised have recently been established; see the paper by Nicole Lemire, Vladimir Popov and Zinovy Reichstein [58] where this problem was posed and settled in the case of algebraically closed ground field and the subsequent papers $[12,13]$ for the treatment of the general case.

However, neither these 'prehistoric' methods, nor the exponential map introduced by Lie and Cartan, nor the more recent approach by Mal'cev and Lazard mentioned above can help with our problem of finding infinitesimal analogues of Borel's theorem. The main point is that there is no obvious way to arrange the transfer to move group commutator to Lie bracket.

Moreover, it turns out that a straightforward transfer of the dominance statement to the case of a semisimple Lie algebra $\mathfrak{g}$ cannot hold because there are Lie polynomials identically zero on $\mathfrak{g}$. Here is a counter-optimistic example: for $\mathfrak{g}=\mathfrak{s l}(2, k)$ ( $k$ is a field of characteristic zero) and $P(X, Y, Z)=[[[[[Z, Y], Y], X], Y],[[[[Z, Y], X], Y], Y]]$, Yuri Razmyslov [73] showed that $P(X, Y, Z) \equiv 0$ on $\mathfrak{g}$.

Of course, it was known well before Borel's theorem that such a phenomenon cannot occur for word maps on semisimple algebraic groups: in characteristic zero this follows from the famous alternative established by Jacques Tits [90], in general from an even earlier paper by Vladimir Platonov [71].
This observation might lead the reader to the conclusion that a road from Borel's theorem towards its eventual infinitesimal analogue cannot look like a freeway paved with classical works in Lie theory, but is rather similar to a rocky mountain road. However, the following theorem proved in our joint paper [3] with Bandman, Gordeev and Plotkin may suggest a more sober viewpoint, where the phenomena similar to Razmyslov's example are considered as sort of potholes to be circumvented.

Theorem 6.2. Let $\mathrm{g}(\mathrm{R}, k)$ be a Chevalley algebra. If $\operatorname{char}(k)=2$, assume that $\mathrm{R}$ does not contain irreducible components of type $\mathrm{C}_{r}, r \geq 1$ (here $\mathrm{C}_{1}=\mathrm{A}_{1}, \mathrm{C}_{2}=\mathrm{B}_{2}$ ).

Suppose $P\left(X_{1}, \ldots, X_{d}\right)$ is not an identity of the Lie algebra $\mathfrak{s l}(2, k)$. Then the induced map $P: \mathfrak{g}(\mathrm{R}, k)^{d} \rightarrow \mathfrak{g}(\mathrm{R}, k)$ is dominant.

Here $\mathrm{R}$ stands for a root system, and $\mathrm{g}(\mathrm{R}, k)$ denotes the Lie algebra over $k$ obtained from the corresponding complex semisimple Lie algebra $\mathfrak{g}(\mathrm{R}, \mathbb{C})$ using its Chevalley basis. In fact, it is the notion of Chevalley basis that allows one to streamline the road from algebraic groups to Lie algebras (in the case they are semisimple). More specifically, one of the crucial tools here is the so-called adjoint quotient, which was also invented by Claude Chevalley and further developed by Tonny Springer and Robert Steinberg [88].

Remark 6.3. We do not know whether the assumption on the polynomial $P$ in Theorem 6.2 can be removed.

Remark 6.4. Theorem 6.2 can be used to reduce, for any given Lie polynomial, the problem of the dominance of the corresponding evaluation map on simple Lie algebras to the case of algebras of types $A_{2}$ and $B_{2}$, see [3].

One can try to pursue the obtained parallel regarding Zariski dominance to the case of Euclidean topology, with an aim to check whether some counterpart of Thom's phenomenon (see Remark 5.5) can occur.

Question 6.5. Do there exist a Lie polynomial $P$ and a compact simple real Lie algebra $g$ such that the image of map (5) is not dense in Euclidean topology?

Remark 6.6. There is much less hope of establishing any relationship between the affirmative answers to Questions 5.2(a) and 6.1(a), in other words, between the surjectivity of the word map $w$ on a semisimple group $G$ (defined with the help of group commutators) and the corresponding polynomial map on the Lie algebras $\mathfrak{g}=\operatorname{Lie}(G)$ induced by the Lie polynomial obtained from $w$ by replacing group commutators with Lie brackets. An explicit example can be found in the paper by Tatiana Bandman and Yuri Zarhin [4].

In light of these remarks, I regret to say that as of now, here an eventual route towards infinitesimal analogues rather looks like a mountain road.

\section{$7 \quad$ Local-global invariants of groups and Lie algebras}

In the last part of this survey, we consider two well-known objects of cohomological nature related to a given group $G$. 
They proved useful in many important problems, some of which are beyond group theory (see my survey [56] and our joint paper with Kang [49] for details). It turned out recently that they naturally appear as parts of certain cohomology of Hopf algebras (called lazy cohomology, see the paper by Pierre Guillot and Christian Kassel [38], or invariant cohomology, see the paper by Pavel Etingof and Shlomo Gelaki [27]). An even more surprising relation, where the two invariants fit together nicely within another invariant with the origin in mathematical physics (the so-called group of braided tensor autoequivalences of the Drinfeld centre of $G$ ), was discovered by Alexei Davydov [23,24].

We are not going to discuss these spectacular achievements here. Our interest is rather in very recent Lie-algebraic analogues of these group-theoretic objects and eventual parallels. (This is an ongoing joint project with Vadim Ostapenko.)

\section{Bogomolov multiplier}

Recall that the Schur multiplier of a group $G$ is defined as the second cohomology group $H^{2}(G, \mathbb{Q} / \mathbb{Z}$ ) (where the action of $G$ on $\mathbb{Q} / \mathbb{Z}$ is trivial).

The Bogomolov multiplier $B(G)$ of a finite group $G$ is defined by

$$
B(G):=\operatorname{ker}\left[H^{2}(G, \mathbb{Q} / \mathbb{Z}) \rightarrow \prod_{A<G \text { abelian }} H^{2}(A, \mathbb{Q} / \mathbb{Z})\right] ;
$$

Fedor Bogomolov showed [9] that in this formula one can replace 'abelian' by 'bicyclic'.

The group $B(G)$ appeared in some algebraic-geometric context: it coincides with the unramified Brauer group of the quotient $V / G$ of a faithful action of $G$ on a complex vector space $V$, and thus is a birational invariant of this variety; this allowed David Saltman [79] to produce first counter-examples to Emmy Noether's problem on the rationality of fields of invariants of permutation groups.

One can also note a recent unexpected application of the Bogomolov multiplier outside group theory: after extending the definition to profinite groups, $B(G)$ can be interpreted in the context of the noncommutative Iwasawa theory (as the kernel of the map $K_{1}\left(\mathbb{Z}_{p}[[G]]\right) \rightarrow K_{1}\left(\mathbb{Q}_{p}[[G]]\right)$, see the paper by Urban Jezernik and Jonatan Sánchez [45]).

One should also mention some recently discovered relations of the Bogomolov multiplier to more conventional group-theoretic problems, in the spirit of Section 3 of the present paper. Namely, in the same paper [45] Jezernik and Sánchez showed that if one fixes an odd prime $p$ and considers the asymptotic behaviour of all finite $p$-groups, the (logarithmically scaled) proportion of wide groups tends to 1 ; the same is true of the proportion of groups with nonzero Bogomolov multiplier. In another paper by Jezernik [44] (joint with Primož Moravec), one can find an interesting link between $B(G)$ and the classical notion of commuting probabilty of $G$, going back to Paul Erdős and Paul Turán.

Let $L$ now be a (finite-dimensional) Lie algebra over a field $k$. Assume for simplicity that $k$ is of characteristic zero. To define an analogue $B(L)$ of the Bogomolov multiplier, one can use another interpretation of $B(G)$ due to Moravec [65]. It is based on the notion of nonabelian exterior square $G \wedge G$. The Schur multiplier is dual to the kernel $M(G)$ of the natural map $G \wedge G \rightarrow[G, G], g \wedge h \mapsto[g, h]$, and $B(G)$ is dual to the quotient $M(G) / M_{0}(G)$, where $M_{0}(G)$ is generated by $g \wedge h$ with commuting $g, h$.

It turns out that this construction can be transferred to Lie algebras; details can be found in recent papers by Zeinab Araghi Rostami, Mohsen Parvizi and Peyman Niroomand $[75,76]$.

A primary goal is to transfer to $B(L)$ as many known properties of $B(G)$ as possible. Whereas the Bogomolov multiplier vanishes on finite simple groups [55] and simple Lie algebras (follows from the results of Peggy Batten's PhD thesis [6] where the vanishing of the Schur multiplier of simple Lie algebras is proven), on the other extreme edge ( $p$ groups/nilpotent Lie algebras) there are nontrivial examples. For instance, in the paper [29] Gustavo Fernández-Alcober and Urban Jezernik showed that the Bogomolov multiplier of a $p$-group can be as large as we wish. In the papers $[75,76]$ mentioned above, one can find examples of finite-dimensional nilpotent Lie algebras with nontrivial Bogomolov multiplier. However, the following question is still open.

Question 7.1. Can the dimension of $B(L)$ be as large as possible?

Further, recall the notion of isoclinism introduced by Philip Hall in the group-theoretic context [40]. Groups $G$ and $H$ are called isoclinic if there are isomorphisms from $G / Z(G)$ to $H / Z(H)$ and from $G /[G, G]$ to $H /[H, H]$ commuting with the commutator map. This notion proved useful in lots of problems, particularly in the classification of finite $p$-groups, and was later on extended to associative rings (see the monograph [54] by Robert Kruse and David Price), to Lie algebras by Kay Moneyhun [64], and more recently, with certain modifications, to more general algebraic structures by Stephen Buckley [15].

Isoclinic objects often share many important properties. In this connection, in our joint paper with Akinari Hoshi and Ming-chang Kang [43] it was conjectured that isoclinic finite groups have the same Bogomolov multiplier. This was proved by Moravec [65] and later strengthened by Fedor Bogomolov and Christian Böhning [10] by showing that if $G$ and $H$ are isoclinic, the orbit varieties $V / G$ and $W / H$ with respect to their faithful actions are stably birationally equivalent.

This leads to the following question.

Question 7.2. Is $B(L)$ invariant under isoclinism of Lie algebras?

In a more conceptual mood, one could try to revisit the results of [45] where it is shown that 'generically' (in some appropriate sense a hint to which was given above), $B(G)$ is nontrivial. Can one translate this statement into the language of Lie algebras?

\section{Shafarevich-Tate groups and algebras}

Let a group $G$ act on itself by conjugation. The ShafarevichTate set is defined with the help of (nonabelian) group cohomology corresponding to this action, by the formula

$$
\amalg(G):=\operatorname{ker}\left[H^{1}(G, G) \rightarrow \prod_{C<G \text { cyclic }} H^{1}(C, G)\right] .
$$

The definition and the name were introduced by Takashi Ono $[68,69]$. The local-global flavour justifies the allusion 
to the object bearing the same name which appeared in the arithmetic-geometric context (related to the action of the absolute Galois group of a number field $K$ on the group $A(\bar{K})$ of $\bar{K}$-points of an abelian $K$-variety $A$ ). The usage of the Cyrillic letter Ш ("Sha") in this notation was initiated by John Cassels because of its appearance as the first letter in the surname of Igor Shafarevich.

Formula (6) admits a more down-to-earth interpretation, attributed in [69] to Marcin Mazur: $\amalg(G)$ can be identified with the collection of all locally inner (=pointwise inner $=$ class preserving) endomorphisms, i.e., $f \in \operatorname{End}(G)$ with the property $f(g)=a^{-1} g a$ (where $a$ depends on $g$ ). Note that any class preserving endomorphism is injective. Hence, if $G$ is finite, it is surjective, and we arrive at the object introduced by William Burnside more than 100 years ago:

$$
\amalg(G) \cong \operatorname{Aut}_{\mathrm{c}}(G) / \operatorname{Inn}(G),
$$

where $\operatorname{Aut}_{\mathrm{c}}(G)$ stands for the group of class-preserving automorphisms of $G$. In particular, this means that if $G$ is finite, $\amalg(G)$ is a group, not just a pointed set. (Ono [69] extended this to the case where $G$ is profinite.)

In my survey [56] (see also the survey by Manoi Yadav [94]), one can find many classes of groups $G$ with trivial $\amalg(G)$ (they are called $\amalg$-rigid there), as well as some interesting examples with nontrivial $\amalg(G)$ (they often give rise to counter-examples to some difficult problems, such as Higman's problem on isomorphism of integral group rings).

Let us now go towards a Lie-algebraic analogue of the Shafarevich-Tate group, taking formula (6) as a starting point. Given a Lie algebra $L$ over a field $k$ (for simplicity assumed to be of characteristic zero), consider the Chevalley-Eilenberg first (adjoint) cohomology $H^{1}(L, L)$. It is well known that it can be identified with the outer derivations $\operatorname{Out}(L):=$ $\operatorname{Der}(L) / \operatorname{ad}(L)$. Recall that $\operatorname{ad}(L)$ is the collection of all inner derivations of $L$ defined by the formula $\operatorname{ad}_{Z}(X)=[Z, X]$. Viewing $\operatorname{Der}(L)$ as a Lie algebra and noticing that $\operatorname{ad}(L)$ is its Lie ideal, we obtain a Lie algebra structure on $\operatorname{Out}(L)$.

Further, define 'locally inner' derivations by

$$
\operatorname{Der}_{\mathrm{c}}(L):=\{D \in \operatorname{Der}(L) \mid(\forall X \in L)(\exists Z \in L) D(X)=[Z, X]\}
$$

(here $Z$ depends on $X$ ).

This notion was introduced by Carolyn Gordon and Edward Wilson [36] (under the name of 'almost inner' derivations) in the differential-geometric context, allowing them to produce a continuous family of isospectral non-isometric compact Riemann manifolds. Recently, the interest in these Lie-algebraic structures was revived in the series of papers by Farshid Saeedi and his collaborators [84-86], and also in the series of papers by Dietrich Burde, Karel Dekimple and Bert Verbecke [16-18].

First, one can note that $\operatorname{Der}_{\mathrm{c}}(L)$ is a Lie subalgebra of $\operatorname{Der}(L)$ [16, Proof of Proposition 2.3], and $\operatorname{ad}(L)$ is a Lie ideal of $\operatorname{Der}_{\mathrm{c}}(L)$.

Definition 7.3. Set $\amalg(L):=\operatorname{Der}_{\mathrm{c}}(L) / \operatorname{ad}(L)$ and call it the Shafarevich-Tate algebra of $L$.

By analogy with the group case, we introduce the following notion (cf. [56]).

Definition 7.4. If $\amalg(L)=0$, we say that $L$ is $\amalg$-rigid.
Here are some parallels between the $\amalg$-rigidity of groups and Lie algebras. If not stated otherwise, the group-theoretic facts mentioned below are taken from the survey [56], where the reader can find the references to the original works, and the Lie-algebraic statements are borrowed from [17].

1. Any simple finite-dimensional Lie algebra $L$ over a field $k$ of characteristic zero is $\amalg$-rigid because $L$ has no outer derivations at all (Whitehead's first lemma, the proof does not use the classification). There are many classes of algebras with no outer derivations, in particular, many complete algebras (with trivial centre and no outer derivations) are known. In the parallel universe of finite groups, it is known that all finite simple groups are Ш-rigid (Walter Feit and Gary Seitz [28], the proof heavily relies on the classification). Note that the situation is different for infinite groups (and may be different for finitedimensional Lie algebras over fields of positive characteristic or infinite-dimensional Lie algebras).

2. The following groups (Lie algebras over a field of characteristic zero) are Ш-rigid: free nilpotent; abelian-bycyclic groups (resp. finite-dimensional Lie algebras with codimension one abelian ideal); extraspecial groups of or$\operatorname{der} p^{2 n+1}$ (resp. Heisenberg Lie algebras $H_{2 n+1}$ ([16, Example 2.6] for $n=1$ ); $p$-groups of order at most $p^{4}$ (resp. nilpotent Lie algebras of dimension at most 4). Note that the list of known classes of $\amalg$-rigid Lie algebras is far shorter than the list of known classes of $\amalg$-rigid groups, and one can continue producing more $\amalg$-rigid Lie algebras, taking $\amalg$-rigid groups as a source for inspiration.

3. On the other hand, many examples of algebras with nonzero $\amalg(L)$ can be found among nilpotent and, more generally, solvable algebras $[16,36,86]$. The dimension of $\amalg(L)$ can be arbitrarily large [36]. In the nilpotent case, a more direct relation between $\amalg(G)$ and $\amalg(L)$ could apparently be formulated, in the spirit of [36].

Here are some open problems.

Question 7.5. Is the Lie algebra $\amalg(L)$ solvable?

Note that for any finite group $G$ it is conjectured that the group $\amalg(G)$ is solvable. The proof of this statement in the paper by Chih-han Sah [78] contains a gap. This was noticed by Masafumi Murai [66] who showed that its validity depends on the Alperin-McKay conjecture.

Question 7.6. Does there exist $L$ such that $\amalg(L)$ is nonabelian?

Note that Sah [78] disproved Burnside's statement [20] and exhibited examples of $p$-groups $G$ with nonabelian $\amalg(G)$, the smallest among them is a group of order $2^{15}$.

Further, since $\amalg(G)$ is known to be an isoclinic invariant according to Yadav [93], it is natural to pose a question in the spirit of Question 7.2.

Question 7.7. Is $\amalg(L)$ invariant under isoclinism of Lie algebras?

Remark 7.8. To conclude, I would like to mention eventual analogues of $\amalg(G)$ and $\amalg(L)$ that one can introduce for other classes of algebras. First of all, this is the class of associative algebras where, given such an algebra $A$, one can 
define the multiplicative $\amalg$ consisting of outer 'locally inner' automorphisms of $A$ as well as the additive $\amalg$ consisting of outer 'locally inner' derivations of $A$. Second, one can consider various generalisations of Lie algebras (Leibniz algebras, Mal'cev algebras, etc.) as well as their counterparts serving as analogues of Lie groups. In the opposite direction, one can enrich a Lie algebra with some additional structure and consider the arising versions of Ш. The first interesting case on this route is the class of Poisson algebras. But this is another story. To obtain insight into eventual parallels among these new objects, one will have to use modern bridges rather than the older ones.

\section{Bibliography}

[1] D. Akhiezer, On the commutator map for real semisimple Lie algebras, Moscow Math. J. 15 (2015), 609-613.

[2] T. Bandman, S. Garion, B. Kunyavskiǔ, Equations in simple matrix groups: algebra, geometry, arithmetic, dynamics, Central Eur. J. Math. 12 (2014), 175-211.

[3] T. Bandman, N. Gordeev, B. Kunyavskiĭ, E. Plotkin, Equations in simple Lie algebras, J. Algebra 355 (2012), 67-79.

[4] T. Bandman, Yu. G. Zarhin, Surjectivity of certain word maps on PS L $(2, \mathbb{C})$ and $S L(2, \mathbb{C})$, Eur. J. Math. 2 (2016), 614-643.

[5] J. Barge, E. Ghys, Cocycles d'Euler et de Maslov, Math. Ann. 294 (1992), 235-265.

[6] P. G. Batten, Multipliers and covers of Lie algebras, PhD Thesis, North Carolina State Univ., 1993.

[7] G. M. Bergman, N. Nahlus, Homomorphisms on infinite direct product algebras, especially Lie algebras, J. Algebra $\mathbf{3 3 3}$ (2011), 67-104.

[8] Y. Billig, V. Futorny, Lie algebras of vector fields on smooth affine varieties, Comm. Algebra 46 (2018), 3413-3429.

[9] F. A. Bogomolov, The Brauer group of quotient spaces of linear representations, Izv. Akad. Nauk SSSR Ser. Mat. 51 (1987), 485-516; English transl. in Math. USSR-Izv. 30 (1988), 455485.

[10] F. Bogomolov, C. Böhning, Isoclinism and stable cohomology of wreath products, in: "Birational Geometry, Rational Curves, and Arithmetic", Simons Symp., Springer, Cham, 2013, pp. 57-76.

[11] A. Borel, On free subgroups of semisimple groups, Enseign. Math. 29 (1983), 151-164.

[12] M. Borovoi, B. Kunyavskii, Stably Cayley semisimple groups, Documenta Math. Extra Volume: Alexander S. Merkurjev's Sixtieth Birthday (2015), 85-112.

[13] M. Borovoi, B. Kunyavskii, N. Lemire, Z. Reichstein, Stably Cayley groups in characteristic zero, Int. Math. Res. Notices 2014, 5340-5397.

[14] G. Brown, On commutators in a simple Lie algebra, Proc. Amer. Math. Soc. 14 (1963), 763-767.

[15] S. M. Buckley, Distributive algebras, isoclinism, and invariant probabilities, in: "Noncommutative Rings and their Applications", Contemp. Math., vol. 634, Amer. Math. Soc., Providence, RI, 2015, pp. 31-52,

[16] D. Burde, K. Dekimpe, B. Verbeke, Almost inner derivations of Lie algebras, J. Algebra Appl. 17 (2018), no. 11, 1850214, $26 \mathrm{pp}$.

[17] D. Burde, K. Dekimpe, B. Verbeke, Almost inner derivations of Lie algebras, II, arXiv:1905.08145. To appear in Intern. J. Algebra Comput.

[18] D. Burde, K. Dekimpe, B. Verbeke, Almost inner derivations of 2-step nilpotent Lie algebras of genus 2, Linear Algebra Appl. 608 (2021), 185-202.

[19] W. Burnside, Theory of Groups of Finite Order, second ed., Cambridge Univ. Press, Cambridge, 1911; reprinted by Dover Publications, New York, 1955.
[20] W. Burnside, On the outer automorphisms of a group, Proc. London Math. Soc. 11 (1913), 40-42.

[21] P.-E. Caprace, K. Fujiwara, Rank-one isometries of buildings and quasi-morphisms of Kac-Moody groups, Geom. Funct. Anal. 19 (2010), 1296-1319.

[22] A. D'Andrea, A. Maffei, Commutators of small elements in compact semisimple groups and Lie algebras, J. Lie Theory $\mathbf{2 6}$ (2016), 683-690.

[23] A. Davydov, Bogomolov multiplier, double class-preserving automorphisms, and modular invariants for orbifolds, J. Math. Phys. 55 (2014), no. 9, 092305, 13 pp.

[24] A. Davydov, Unphysical diagonal modular invariants, J. Algebra 446 (2016), 1-18.

[25] D. Ž. Đoković, T.-Y. Tam, Some questions about semisimple Lie groups originating in matrix theory, Canad. J. Math. 46 (2003), 332-343.

[26] E. W. Ellers, N. Gordeev, On the conjectures of J. Thompson and O. Ore, Trans. Amer. Math. Soc. 350 (1998), 3657-3671.

[27] P. Etingof, S. Gelaki, Invariant Hopf 2-cocycles for affine algebraic groups, Int. Math. Res. Not. IMRN 2020, no. 2, 344-366.

[28] W. Feit, G. M. Seitz, On finite rational groups and related topics, Illinois J. Math. 33 (1989), 103-131.

[29] G. A. Fernández-Alcober, U. Jezernik, Bogomolov multipliers of p-groups of maximal class, Quart. J. Math. 71 (2020), 121-138.

[30] E. Fink, A. Thom, Palindromic words in simple groups, Internat. J. Algebra Comput. 25 (2015), 439-444.

[31] W. B. Fite, On metabelian groups, Trans. Amer. Math. Soc. 3 (1902), 331-353.

[32] N. L. Gordeev, B. È. Kunyavskii, E. B. Plotkin, Word maps and word maps with constants of simple algebraic groups, Doklady Akad. Nauk 471 (2016), no. 2, 136-138; English transl. in Doklady Math. 94 (2016), 632-634.

[33] N. Gordeev, B. Kunyavskii, E. Plotkin, Word maps, word maps with constants and representation varieties of one-relator groups, J. Algebra 500 (2018), 390-424.

[34] N. Gordeev, B. Kunyavskii, E. Plotkin, Word maps on perfect algebraic groups, Intern. J. Algebra Comput. 28 (2018), 14871515.

[35] N. L. Gordeev, B. È. Kunyavskii, E. B. Plotkin, Geometry of word equations in simple algebraic groups over special fields, Uspekhi Mat. Nauk 73 (2018), no. 5, 3-52; English transl. in Russian Math. Surveys 73 (2018), 753-796.

[36] C. S. Gordon, E. N. Wilson, Isospectral deformations of compact solvmanifolds, J. Diff. Geom. 19 (1984), 241-256.

[37] J. Grabowski, Isomorphisms and ideals of the Lie algebras of vector fields, Invent. Math. 50 (1978/79), 13-33.

[38] P. Guillot, C. Kassel, Cohomology of invariant Drinfeld twists on group algebras, Int. Math. Res. Not. IMRN 2010, no. 10, 1894-1939.

[39] R. M. Guralnick, Expressing group elements as commutators, Rocky Mountain J. Math. 10 (1980), 651-654.

[40] P. Hall, The classification of prime-power groups, J. reine angew. Math. 182 (1940), 130-141.

[41] R. Hirschbühl, Commutators in classical Lie algebras, Linear Algebra Appl. 142 (1990), 91-111.

[42] K. H. Hofmann, S. A. Morris, The Lie Theory of Connected ProLie Groups. A Structure Theory for Pro-Lie Algebras, Pro-Lie Groups, and Connected Locally Compact Groups, EMS Tracts Math., vol. 2, Eur. Math. Soc., Zürich, 2007.

[43] A. Hoshi, M.-C. Kang, B. Kunyavskii, Noether's problem and unramified Brauer groups, Asian J. Math. 17 (2013), 689-713.

[44] U. Jezernik, P. Moravec, Universal commutator relations, Bogomolov multipliers, and commuting probability, J. Algebra 428 (2015), 1-25.

[45] U. Jezernik, J. Sánchez, Irrationality of generic quotient varieties via Bogomolov multipliers, arXiv:1811:01851.

[46] D. A. Jordan, On the ideals of a Lie algebra of derivations, J. London Math. Soc. 33 (1986), 33-39. 
[47] V. G. Kac, Simple irreducible graded Lie algebras of finite growth, Izv. Akad. Nauk SSSR Ser. Mat. 32 (1968), 1323-1367; English transl. in Math. USSR-Izv. 2 (1968), 1271-1311.

[48] A. Kanel-Belov, B. Kunyavskiı̌, E. Plotkin, Word equations in simple groups and polynomial equations in simple algebras, Vestnik St. Petersburg Univ. Math. 46 (2013), no. 1, 3-13.

[49] M. Kang, B. Kunyavskii, The Bogomolov multiplier of rigid finite groups, Arch. Math. (Basel) 102 (2014), 209-218.

[50] L.-C. Kappe, R. F. Morse, On commutators in groups, in: "Groups St. Andrews 2005. Vol. 2", London Math. Soc. Lecture Note Ser., vol. 340, Cambridge Univ. Press, Cambridge, 2007, pp. 531-558

[51] E. I. Khukhro, p-Automorphisms of Finite p-Groups, Cambridge Univ. Press, 1998.

[52] A. Klyachko, A. Thom, New topological methods to solve equations over groups, Algebr. Geom. Topol. 17 (2017), 331-353.

[53] H. Kraft, On the Lie algebras of vector fields, Lecture notes, 2017, available at https://kraftadmin.wixsite.com/hpkraft.

[54] R. L. Kruse, D. T. Price, Nilpotent Rings, Gordon and Breach Science Publishers, New York-London-Paris, 1969.

[55] B. Kunyavskiı̌, The Bogomolov multiplier of finite simple groups, in: "Cohomological and Geometric Approaches to Rationality Problems", Progr. Math., vol. 282, Birkhäuser Boston, Inc., Boston, MA, 2010, pp. 209-217.

[56] B. Kunyavski1, Local-global invariants of finite and infinite groups: around Burnside from another side, Expo. Math. 31 (2013), 256-273.

[57] B. Kunyavskiǔ, A. Regeta, Bracket width of simple Lie algebras, in preparation.

[58] N. Lemire, V. L. Popov, Z. Reichstein, Cayley groups, J. Amer. Math. Soc. 19 (2006), 921-967.

[59] M. Leuenberger, A. Regeta, Vector fields and automorphism groups of Danielewski surfaces, Int. Math. Res. Not. IMRN 2020, rnaa189.

[60] M. W. Liebeck, E. A. O'Brien, A. Shalev, P. H. Tiep, The Ore conjecture, J. Europ. Math. Soc. 12 (2010), 939-1008.

[61] J. Malkoun, N. Nahlus, Commutators and Cartan subalgebras in Lie algebras of compact semisimple Lie groups, J. Lie Theory 27 (2017), 1027-1032.

[62] G. Malle, The proof of Ore's conjecture [after Ellers-Gordeev and Liebeck-O'Brien-Shalev-Tiep], Astérisque 361 (2014), exp. no. 1069, 325-348.

[63] MathOverflow discussion, mathoverflow.net/questions/44269.

[64] K. Moneyhun, Isoclinisms in Lie algebras, Algebras Groups Geom. 11 (1994), 9-22.

[65] P. Moravec, Unramified Brauer groups of finite and infinite groups, Amer. J. Math. 134 (2012), 1679-1704.

[66] M. Murai, Blocks of normal subgroups, automorphisms of groups, and the Alperin-McKay conjecture, Kyoto J. Math. 54 (2014), 199-238.

[67] A. Muranov, Finitely generated infinite simple groups of infinite commutator width, Internat. J. Algebra Comput. 17 (2007), 607659.

[68] T. Ono, A note on Shafarevich-Tate sets for finite groups, Proc. Japan Acad. Ser. A Math. Sci. 74 (1998), no. 5, 77-79.

[69] T. Ono, "Shafarevich-Tate sets" for profinite groups, Proc. Japan Acad. Ser. A Math. Sci. 75 (1999), no. 6, 96-97.

[70] O. Ore, Some remarks on commutators, Proc. Amer. Math. Soc. 2 (1951), 307-314.

[71] V. P. Platonov, Linear groups with identical relations, Dokl. Akad. Nauk BSSR 11 (1967), 581-582 (in Russian).

[72] M. M. Postnikov, Lie Groups and Lie Algebras, "Lectures in Geometry. Semester V", Nauka, Moscow, 1982; English transl.: Mir, Moscow, 1986.

[73] Yu. P. Razmyslov, The existence of a finite basis for the identities of the matrix algebra of order two over a field of characteristic zero, Algebra Logika 12 (1973), no. 1, 83-113; English transl. in: Algebra Logic 12 (1973), 47-63.
[74] R. Ree, Commutators in semi-simple algebraic groups, Proc. Amer. Math. Soc. 15 (1964), 457-460.

[75] Z. A. Rostami, M. Parvizi, P. Niroomand, The Bogomolov multiplier of Lie algebras, Hacet. J. Math. Stat. 49 (2020), 11901205.

[76] Z. A. Rostami, M. Parvizi, P. Niroomand, Bogomolov multiplier and the Lazard correspondence, Comm. Algebra 48 (2020), 1201-1211.

[77] A. N. Rudakov, Automorphism groups of infinite-dimensional simple Lie algebras, Izv. Akad. Nauk SSSR Ser. Mat. 33 (1969), 748-764; English transl. Math. USSR Izv. 3 (1969), 707-722.

[78] C.-H. Sah, Automorphisms of finite groups, J. Algebra 10 (1968), 47-68; add. ibid. 44 (1977), 573-575.

[79] D. J. Saltman, Noether's problem over an algebraically closed field, Invent. Math. 77 (1984), 71-84.

[80] M. Schlichenmaier, Krichever-Novikov Type Algebras. Theory and Applications, De Gruyter Stud. Math., vol. 53, De Gruyter, Berlin, 2014.

[81] D. Segal, Words: Notes on Verbal Width in Groups, London Math. Soc. Lecture Note Ser., vol. 361, Cambridge Univ. Press, Cambridge, 2009.

[82] A. Shalev, Commutators, words, conjugacy classes and character methods, Turkish J. Math. 31 (2007), 131-148.

[83] A. Shalev, Some results and problems in the theory of word maps, in: "Erdös Centennial" (L. Lovász, I. Ruzsa, V. T. Sós, D. Palvolgyi, Eds.), Bolyai Soc. Math. Studies, vol. 25, Springer, 2013.

[84] S. Sheikh-Mohseni, F. Saeedi, Camina Lie algebras, Asian-Eur. J. Math. 11 (2018), no. 5, 1850063, 11 pp.

[85] S. Sheikh-Mohseni, F. Saeedi, On the dimension of a special subalgebra of derivations of nilpotent Lie algebras, Bull. Iranian Math. Soc. 43 (2017), 79-93.

[86] S. Sheikh-Mohseni, F. Saeedi, M. Badrkhani Asl, On special subalgebras of derivations of Lie algebras, Asian-Eur. J. Math. 8 (2015), no. 2, 1550032, 12 pp.

[87] T. Siebert, Algebras of derivations and affine algebraic geometry over fields of characteristic 0, Math. Ann. 305 (1996), 271-286.

[88] T. A. Springer, R. Steinberg, Conjugacy classes, in: "Seminar on Algebraic Groups and Related Finite Groups", Lecture Notes Math., vol. 131, Springer-Verlag, Berlin-Heidelberg-New York, 1970, pp. 167-266.

[89] A. Thom, Convergent sequences in discrete groups, Canad. Math. Bull. 56 (2013), 424-433.

[90] J. Tits, Free subgroups in linear groups, J. Algebra 20 (1972), 250-270.

[91] A. Weinstein, Poisson geometry, in: "Symplectic Geometry", Differential Geom. Appl. 9 (1998), 213-238.

[92] H. Weyl, The Classical Groups. Their Invariants and Representations, Princeton Univ. Press, Princeton, NJ, 1939.

[93] M. K. Yadav, On automorphisms of some finite p-groups, Proc. Indian Acad. Sci. (Math. Sci.) 118 (2008), 1-11.

[94] M. K. Yadav, Class preserving automorphisms of finite pgroups: A survey, in: "Groups St Andrews 2009 in Bath. Vol. 2", London Math. Soc. Lecture Note Ser., vol. 388, Cambridge Univ. Press, Cambridge, 2011, pp. 569-579.

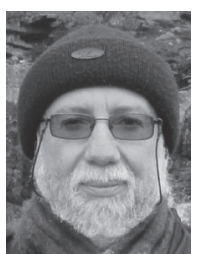

Boris Kunyavskii [kunyav@gmail.com] is a professor of mathematics at Bar-Ilan University (Ramat Gan, Israel). His field of expertise is algebra. His current research interests are concentrated around algebraic groups, group theory and Lie algebras. 